\title{
Continuous Glucose Monitoring in 2016
}

\author{
Bruce W. Bode ${ }^{1}$ and Tadej Battelino ${ }^{2,3}$
}

\section{Introduction}

D ESPITE THE PERVASIVE BOOM in continuous glucose monitoring (CGM) research and clinical practice it seems that finally its real clinical target is coming into focus: the glycemic variability $(\mathrm{GV})$. It took diabetologists a good decade to incorporate the overwhelmingly clear message of the Diabetes Control and Complications Trial (DCCT) trial into routine clinical practice and we do not look likely to increase our enthusiasm and appetite for incorporating new concepts like GV any faster nowadays. Our relentless reluctance of accepting new clinical paradigms may be described as healthy professional conservatism, however, it becomes a pity when opportunities for better diabetes care are missed.

CGM recently gained concensus to be used for insulin treatment decision making, and the Food and Drug Administration (FDA) cleared the first device for hybrid closed-loop insulin delivery. Impressively, this highly stringent regulatory body evaluated the existing evidence and understood the importance of its message.

This review therefore focuses on the likely most important clinical target of the CGM technology-the GV.

\author{
Key Articles Reviewed for the Article \\ Glucose variability: timing, risk analysis, and relationship to hypoglycemia in \\ diabetes \\ Kovatchev B, Cobelli $C$ \\ Diabetes Care 2016; 39: 502-510 \\ Continuous glucose monitoring in patients with type 1 diabetes \\ using insulin injections \\ Foster NC, Miller KM, Tamborlane MV, Bergenstal RM, and Beck RW; \\ for the TID Exchange Clinic Network \\ Diabetes Care 2016; 39: e81-82.
}

Nocturnal hypoglycemia and physical activity in children with diabetes: new insights by continuous glucose monitoring and accelerometry

Bachmann S, Hess M, Martin-Diener E, Denhaerynck K, Zumsteg $U$

Diabetes Care 2016; 39: e95-96.

Use of glucose rate of change arrows to adjust insulin therapy among individuals with type 1 diabetes who use continuous glucose monitoring

Pettus J, Edelman SV

Diabetes Technol Ther 2016; 18 Suppl 2: S234-242.

\footnotetext{
${ }^{1}$ Atlanta Diabetes Associates, Atlanta, GA.

${ }^{2}$ UMC-University Children's Hospital Ljubljana, Slovenia.

${ }^{3}$ Faculty of Medicine, University of Ljubljana, Ljubljana, Slovenia.
} 


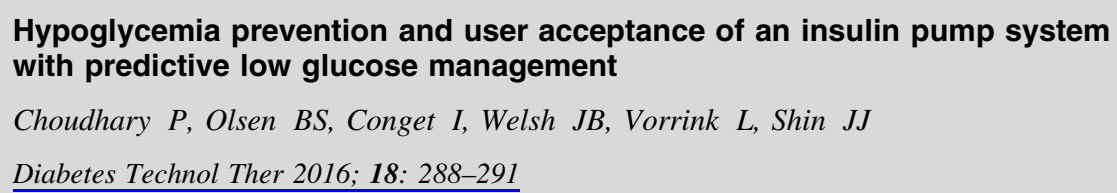

Effects of daily glucose fluctuations on the healing response to everolimus-eluting stent implantation as assessed using continuous glucose monitoring and optical coherence tomography

Kuroda M, Shinke T, Otake H, Sugiyama D, Takaya T, Takahashi H, Terashita D, Uzu K, Tahara N, Kashiwagi D, Kuroda K, Shinkura Y, Nagasawa Y, Sakaguchi K, Hirota $Y$, Ogawa $W$, Hirata $K$

Cardiovasc Diabetol 2016; 15: 79

Glucose variability in a 26-week randomized comparison of mealtime treatment with rapid-acting insulin versus GLP-1 agonist in participants with type 2 diabetes at high cardiovascular risk

Jeffrey L. Probstfield, Irl B. Hirsch, Barry R. Davis, Andrew Ahmann, Richard Bergenstal, Matthew Gilbert, Connie Kingry, Dorrine Khakpour, Dejian Lai, Sarah L. Pressel, Kelley R. Branch, Matthew Riddle, Kevin D. O'Brien; on behalf of the FLAT-SUGAR Trial Investigators

Diabetes Care 2016; 39: 973-981

\section{Glucose variability: timing, risk analysis, and relationship to hypoglycemia in diabetes}

Kovatchev $B^{1}$, Cobelli $C^{2}$

${ }^{I}$ Center for Diabetes Technology, University of Virginia, Charlottesville, VA; ${ }^{2}$ Department of Information Engineering, University of Padova, Padova, Italy

Diabetes Care 2016; 39: 502-510

\section{Aims}

To propose standardization of blood glucose (BG) variability $(\mathrm{GV})$ measurement and restructure the evaluation of its two most important components: amplitude and duration/timing.

\section{Methods}

Methodical analysis of causes, metrics, and clinical manifestations of $\mathrm{GV}$, with a particular emphasis on quantifying the amplitude and the timing/duration of glucose fluctuations and their relevance to underlying disease processes and patients' disease-related behavior. Four case scenarios were studied and different calculations of GV compared in relation to predicting extreme $\mathrm{BG}$ variability.

\section{Results}

GV metrics that do not symmetrize BG readings (SD of BG, $\mathrm{M}$-value, and mean average glucose excursions (MAGE)) are predictive primarily of hyperglycemia and fail to predict future hypoglycemic episodes, similarly to that of average BG and $\mathrm{HbAlc}$, as already described in the Diabetes Control and Complications Trial (DCCT), where only about $7 \%$ of severe hypoglycemic episodes can be accounted for by HbAlc. The average daily risk range (ADRR) symmetrize the $\mathrm{BG}$ scale and thus can predict both hypo- and hyperglycemia. The low blood glucose index (LBGI) and high blood glucose index
(HBGI) predict the risk of hypo- and hyperglycemia separately, concentrating only on the more relevant part of the BG readings scale.

\section{Conclusions}

GV has richer information content than just average glucose (HbA1c), however, its quantitative assessment is not straightforward because of its two components: amplitude and duration/timing. Deviations toward hypoglycemia are much bigger in magnitude when compared to deviations toward hyperglycemia and symmetrization or separate measures for hypo- and hyperglycemia are needed in order to accurately predict extreme BG fluctuations. Assessment of temporal characteristics of GV benefits from mathematical computations that go beyond basic arithmetic and is essential with continuous glucose monitoring (CGM).

\section{Comment}

This educational paper (1) aims to describe in a diabetologist-appropriate language the nature and usefulness of GV, illustrating it with clinical scenarios. Particular stress is placed on the incorporation of the duration/timing of glucose fluctuations in clinical assessment, which need mathematical erudition for accurate evaluation. All clinicians, but diabetologists in particular, should by now be ready to accept appropriate expertise and technology helping us to better care for people with diabetes. Closed-loop insulin delivery would not be possible without real-time assessment of $\mathrm{GV}$, and fitting automated action based on it.

A recent pro-GV paper (2) summarizes the ample evidence for a biological rationale endorsing the concept of $\mathrm{GV}$ as an important risk factor directly involved in the pathogenesis of the vascular complications of diabetes. A large randomized study over many years with hard 
outcome variables is needed to finally prove the real clinical importance of GV; however, current evidence support the notion that $\mathrm{GV}$ is dangerous and should be a primary treatment target, despite arguments in a recent anti-GV paper (3) that other diabetes markers may be more relevant. It seems predictable that our treatment strategies in diabetes will be quite different in the foreseeable future once we understand the importance of $\mathrm{GV}$.

\section{Continuous glucose monitoring in patients with type 1 diabetes using insulin injections}

Foster $N C^{1}$, Miller $K M^{1}$, Tamborlane $M V^{2}$, Bergenstal $R M^{3}$, and Beck $R W^{l}$; for the T1D Exchange Clinic Network

${ }^{1}$ Jaeb Center for Health Research, Tampa, FL; ${ }^{2}$ Yale University School of Medicine, New Haven, CT; ${ }^{3}$ International Diabetes Center at Park Nicollet, Minneapolis, MN

Diabetes Care 2016; 39: e81-82.

\section{Aims}

To evaluate the use of CGM in patients with type 1 diabetes (T1D) using multiple daily injections (MDI) as treatment modality.

\section{Methods}

The T1D Exchange registry database was used to investigate the influence of CGM use on HbA1c in patients using multiple daily injections. CGM use was defined as real-time diabetes management with a CGM device during the 30 days prior to the clinic visit.

\section{Results}

From the registry, 17,731 participants had suffered T1D of $>1$ year duration and had had a clinic visit between June 2014 and October 2015. Injections alone were used by $6222(35 \%)$, $8783(50 \%)$ used pump alone, 2316 (13\%) used a pump with CGM (58\% used Dexcom), and 410 (2\%) used injections with CGM (97\% used Dexcom). The median number of short-acting insulin boluses was three per day (interquartile range 3,4 ), and was the same with or without CGM. Mean HbA1c with CGM was similar in injection and pump users $(7.6 \% \pm 1.3 \%$ vs. $7.7 \% \pm 1.1 \%)$ and lower in CGM users than in non-CGM users: in the pump group $(8.3 \% \pm 1.5 \%$, adjusted $P<0.001)$ and in the injection group $(8.8 \% \pm 1.9 \%$, adjusted $P<0.001)$, both in youth and adults.

\section{Conclusion}

This analysis of T1D Exchange registry data demonstrated an association of CGM use with lower HbAlc, irrespective of the insulin delivery method, even after adjustment for several confounding factors. Interestingly, CGM users on MDI therapy had HbA1c levels comparable to those using CGM with an insulin pump. A potential limitation of the study is the possibility that CGM users had lower HbAlc also for other reasons not investigated in this report. Nevertheless, the results imply that CGM can be helpful for patients on MDI therapy across all age groups.

\section{Comment}

The results of this important report (4) are consistent with the limited data from the adult part of the Juvenile Diabetes Research Foundation (JDRF) CGM randomized controlled trial: nine MDI/CGM users had a comparable HbA1c improvement to 41 pump/CGM users $(-0.54 \%$ vs. $-0.50 \%)(5)$. However, this interesting observation has not been investigated in a randomized controlled trial. Several ongoing studies are investigating the effect of CGM in MDI users (6), however none with a randomized comparison to the CGM in pump users. Finally, glucose variability rather than $\mathrm{HbA} 1 \mathrm{c}$ may be the most clinically important primary outcome measure for trials comparing different insulin delivery modalities in association with the use of CGM.

\section{Nocturnal hypoglycemia and physical activity in children with diabetes: new insights by continuous glucose monitoring and accelerometry}

Bachmann $S^{1}$, Hess $M^{1}$, Martin-Diener $E^{2}$, Denhaerynck $K^{3}$, Zumsteg $U^{1}$

${ }^{1}$ Pediatric Endocrinology and Diabetology, University Children's Hospital Basel, Basel, Switzerland; ${ }^{2}$ Institute for Social and Preventive Medicine, University of Zurich, Zurich, Switzerland; ${ }^{3}$ Institute of Nursing Science, Basel, Switzerland

Diabetes Care 2016; 39: e95-96.

\section{Aims}

Detection and prevention of nocturnal hypoglycemia can improve daytime performance and avert lack of awareness of hypoglycemia. This study aimed at evaluating the frequency and duration of, and the risk factors for nocturnal hypoglycemia in children with T1D.

\section{Methods}

The influence of physical activity on nocturnal hypoglycemia was studied by combining retrospective CGM (iPro, Medtronic Inc.) with accelerometry. Nocturnal hypoglycemia was defined at a glucose level $<3.7 \mathrm{mmol} / \mathrm{L}$. Moderateto-vigorous physical activity (MVPA) was defined as $>2296$ counts per minute and vigorous physical activity (VPA) as $>4012$ counts per minute.

\section{Results}

Data from 51 subjects (29 male, aged 2-17 years; HbA1c $8.1 \% \pm 1.5 \%[65.4 \pm 16.4 \mathrm{mmol} / \mathrm{mol}] ; 36$ on MDI) from a total of 60 enrolled children with T1D for $>6$ months were analyzed. In total, 128 periods of nocturnal hypoglycemia (duration from 10 to $665 \mathrm{~min}$ ) were recorded; only 8 were reported symptomatic. One or more hypoglycemia occurred during 97 nights $(32.7 \%)$. Of all episodes, 36\% lasted $<1 \mathrm{~h}$, $34 \% 1-3 \mathrm{~h}, 24 \% 3-6 \mathrm{~h}$, and $6 \%>6 \mathrm{~h}$. One hour of daytime MVPA increased the risk of nocturnal hypoglycemia by $58 \%$ $(P=0.009)$ and 1 hour of VPA by $82 \%(P=0.01)$. Bedtime glucose $<6 \mathrm{mmol} / \mathrm{L}$ increased the risk of nocturnal hypoglycemia by 2.5 -fold $(P<0.001)$. Hypoglycemia frequency was negatively correlated to HbA1c $(-0.32, P=0.022)$, but 
not associated with age, insulin dose, treatment modality (MDI vs. pump), or diabetes duration.

\section{Conclusion}

Nocturnal hypoglycemia in children with diabetes is mostly asymptomatic, often prolonged, and more frequent after daytime physical activity and at lower bedtime blood glucose.

\section{Comment}

This largely confirmatory observational study (7) uses retrospective CGM for reinforcing the old paradigm of feeding carbohydrates for prevention of hypoglycemia. Interestingly, hyperglycemia and glucose variability are not even mentioned, despite the solid data confirming their deleterious effect on the developing brain. Additionally, the potential benefit of real-time CGM is not discussed. The investigated group of children and adolescents with T1D had an average HbA1c above the median of the large International Society for Pediatric and Adolescent Diabetes (ISPAD) SWEET database and the rate of detected hypoglycemia considerably exceeded recent reports of the German DPV database (8). Particularly worrying is the finding that bedtime blood glucose $<6 \mathrm{mmol} / \mathrm{L}$ doubles the rate of nocturnal hypoglycemia. What level of bedtime glycaemia is then recommended? If we sincerely aim at improving metabolic control in children and adolescents with T1D, it is highly likely that we will have to advise our patients and their families to go beyond feeding carbohydrates and maintaining supraphysiological bedtime blood glucose levels. Indeed, the use of diabetes technology in routine clinical care is associated with reducing HbAlc simultaneously with reducing the rate of hypoglycemia (9).

\section{Use of glucose rate of change arrows to adjust insulin therapy among individuals with type 1 diabetes who use continuous glucose monitoring}

Pettus $\mathrm{J}^{1}$, Edelman $S V^{1,2}$

${ }^{1}$ University of California San Diego, San Diego, CA;

${ }^{2}$ Veterans Affairs Medical Center, San Diego, CA

Diabetes Technol Ther 2016; 18 Suppl 2: S234-242.

\section{Aims}

To compare utilization of CGM focusing on the glucose trend arrow to adjust insulin dosing in T1D between multiple daily insulin injections (MDI) and insulin pump users.

\section{Methods}

An online survey with 70 multiple-choice questions was available in the United States between May 28 and August 26, 2013, using SurveyGizmo (Boulder, CO). Diabetes health-care teams were invited to recruit patients with T1D using (average $\geq 6$ days/week) the Dexcom CGM system (Dexcom, Inc., San Diego, CA), providing voluntary participants with a web link to the survey. The survey included clinical scenarios and covered six segments: patient characteristics; general CGM use; hypoglycemia prevention and management; hyperglycemia pre- vention and management; insulin dosing adjustments (both for meals and correction boluses); and real-time use versus retrospective analysis. Specific questions in illustrated clinical scenarios were targeted to glucose trend arrow utilization. Study outcomes included general CGM use, overall response to CGM, and use of glucose trend arrows to adjust insulin therapy.

\section{Results}

The survey was completed by 222 individuals with T1D from 22 U.S. states: $166(75 \%)$ used insulin pumps and 56 (25\%) used MDI. The mean age for pump users was $45 \pm 14$ years, duration of diabetes $22 \pm 13$ years, $51 \%$ were male, and the self-reported $\mathrm{HbA} 1 \mathrm{c}$ was $6.8 \% \pm 0.8 \%$. The mean age for MDI users was $48 \pm 13$ years, duration of diabetes $20 \pm 14$ years, $39 \%$ were male, and the self-reported $\mathrm{HbA1c}$ was $6.8 \% \pm 0.9 \%$ (all non significant (NS)). Most (97\% pump, 98\% MDI) reported education after high school. More than $80 \%$ of participants from both groups reported using CGM "all" or "most of the time," with three-quarters using it for $>1$ year. Almost fourfifths in both groups reported a decrease in severe hypoglycemia and a reduction in HbAlc during the CGM use. Roughly one half of both groups reported use of CGM trend arrows for insulin dosing. CGM users in the MDI group reported significantly larger reductions of insulin doses when trend arrows pointed to decreasing or rapidly decreasing glucose concentration as compared to CGM users in the pump group.

\section{Conclusion}

The use of CGM was reported to be associated with an improved glycemic control. The majority reported using trend arrows to make multiple and more substantial changes in their insulin dosages than the usually recommended $10 \%-$ $20 \%$ adjustments.

\section{Comment}

This survey (10) provides an interesting insight into daily routine use of CGM in both MDI and pump users, with a significant inherent limitation being the use of selfreported data. The most significant finding is the reported frequency and aggressiveness of insulin dose modification based on glucose concentration trend arrows. Patients' reported daily practice exceeded standard recommendations and reportedly resulted in an improved mean glycaemia with a concomitant reduction in hypoglycemia. Not surprisingly, these practice-derived patient actions narrowly resemble insulin administration strategies in closed-loop insulin delivery algorithms (11): rapid and frequent insulin dosing adjustments result in more time within the glycemic target. Novel clinical guidelines for the utilization of CGM, with a particular emphasis on glucose concentration trend arrows, based on recent data, are urgently needed.

\section{Hypoglycemia prevention and user acceptance of an insulin pump system with predictive low glucose management}

Choudhary $P^{1}$, Olsen $\mathrm{BS}^{2}$, Conget $\mathrm{I}^{3}$, Welsh $\mathrm{JB}^{4}$, Vorrink $L^{5}$, Shin $J^{4}$ 
${ }^{1}$ King's College London, London, UK; ${ }^{2}$ Herlev Hospital, Herlev, Denmark; ${ }^{3}$ Diabetes Unit, Clinic and University Hospital, Barcelona, Spain; ${ }^{4}$ Medtronic International Trading Sárl, Tolochenaz, Switzerland, ${ }^{5}$ Medtronic, Inc., Northridge, CA

Diabetes Technol Ther 2016; 18: 288-291.

This manuscript is also discussed in chapter on Insulin Pumps, page S-19.

\section{Aims}

To conduct a user evaluation study estimating the ability of predicted low glucose suspend in a sensor-augmented insulin pump to prevent hypoglycemia and its acceptability to users.

\section{Methods}

Forty participants with T1D (24 adults, 16 children), knowledgeable in sensor-augmented pump therapy were enrolled in three European centers. Individualized targets for the "low limit threshold" between 50 and $80 \mathrm{mg} / \mathrm{dL}(2.8-4.5$ $\mathrm{mmol} / \mathrm{L}$ ) were set in the MiniMed 640G (Medtronic Inc.) system, as well as individualized settings for alerts and threshold for the SmartGuard system. The SmartGuard system suspends insulin delivery when the glucose level is predicted to drop below $20 \mathrm{mg} / \mathrm{dL}(1.1 \mathrm{mmol} / \mathrm{L})$ above the preset low limit threshold within the next $30 \mathrm{~min}$. Basal insulin automatically resumed once the glucose value was at least $20 \mathrm{mg} / \mathrm{dL}(1.1$ $\mathrm{mmol} / \mathrm{L}$ ) above the preset low limit threshold and predicted to be at least $40 \mathrm{mg} / \mathrm{dL}(2.2 \mathrm{mmol} / \mathrm{L})$ above it within $30 \mathrm{~min}$, and insulin delivery has been suspended for at least $30 \mathrm{~min}$. The system was used for four weeks. Performance of the system was evaluated retrospectively using downloaded pump and sensor data. User acceptance was evaluated with questionnaires.

\section{Results}

Thirty-nine participants (55\% male) completed the study. The mean age was $31.7 \pm 17.1$ years, diabetes duration $17.2 \pm 13.3$ years, $\mathrm{HbA} 1 \mathrm{c}$ level $7.6 \% \pm 0.9 \% \quad(59.6 \pm 9.5$ $\mathrm{mmol} / \mathrm{mol}$ ), and body mass index (BMI) $23.5 \pm 4.0 \mathrm{~kg} / \mathrm{m}^{2}$. The predictive low glucose suspend feature was used for an average of $29.4 \pm 5.0$ days, with 2322 suspends before low events, a frequency of 2.1 per subject per day. The overall duration of a suspend before a low event was $56.4 \pm 9.6 \mathrm{~min}$ (median, 57.9 [interquartile range (IQR), 48.8-63.6] $\mathrm{min}$ ), daytime being significantly shorter than nighttime $(50.2 \pm 10.6$ vs. $69.0 \pm 10.8 \mathrm{~min} ; P<0.0001)$. The mean subsequent glucose concentration nadir was $71.8 \pm 5.2 \mathrm{mg} / \mathrm{dL}(4.0 \pm 0.3 \mathrm{mmol} / \mathrm{L})$ (median 71.8 (IQR, 67.7-76.2) mg/dL $(4.0[3.8 \pm 4.2] \mathrm{mmol} /$ L). Nadir glucose values of $\leq 60 \mathrm{mg} / \mathrm{dL}(\leq 3.3 \mathrm{mmol} / \mathrm{L})$ were observed in $356(15.3 \%)$ of the predictive suspensions, with a mean duration of $36.1 \pm 23.6 \mathrm{~min}$, and the median (IQR) duration of $30(20-45) \mathrm{min}$. Nadir BG values of $\leq 50 \mathrm{mg} / \mathrm{dL}$ ( $\leq 2.8 \mathrm{mmol} / \mathrm{L}$ ) were observed in $207(8.9 \%)$ of the predictive suspensions, with a mean duration of 25.3-20.8 min, and the median (IQR) duration of 20 (10-35) min. Mean and median responses on the 7-point scale questionnaires were between 6 and 7 , favoring the use of suspend before low feature.

\section{Conclusion}

Automatic predictive insulin pump suspension can prevent hypoglycemia without significantly increasing hyperglycemia.

\section{Comment}

In this short observational study (12) the absence of a control group precludes conclusions on efficacy, and the subset of false-positive events cannot be estimated as low blood glucose was not confirmed by a blood glucose measurement. However, as nadir values below $60 \mathrm{mg} / \mathrm{dL}$ were at $15 \%$, it does appear that most hypoglycemia was indeed attenuated or prevented. This comes at a cost of moderate hyperglycemia, which needs more attention and discussion, as most of the damage to the central nervous system, particularly during development, comes from hyperglycemia rather than hypoglycemia (13). Particularly, imaging observations elucidate the fact that hyperglycemia affects structural changes in the developing brains of children and also in the more mature brains of adults. The shift of almost religious focus on hypoglycemia towards hyperglycemia in the clinical environment will take a lot of effort and benevolence to bring about.

\section{Effects of daily glucose fluctuations on the healing response to everolimus-eluting stent implantation as assessed using continuous glucose monitoring and optical coherence tomography}

Kuroda $M^{1}$, Shinke $T^{2}$, Otake $H^{1}$, Sugiyama $D^{2}$, Takaya $T^{l}$, Takahashi $H^{1}$, Terashita $D^{1}$, Uzu $K^{1}$, Tahara $N^{l}$, Kashiwagi $D^{1}$, Kuroda $K^{1}$, Shinkura $Y^{1}$, Nagasawa $Y^{1}$, Sakaguchi $K^{3}$, Hirota $Y^{3}$, Ogawa $W^{3}$, Hirata $K^{1}$

${ }^{I}$ Division of Cardiovascular Medicine, Department of Internal Medicine, Kobe University Graduate School of Medicine, Kobe, Japan; ${ }^{2}$ Department of Preventive Medicine and Public Health, School of Medicine, Keio University, Tokyo, Japan, ${ }^{3}$ Division of Diabetes and Metabolism, Department of Internal Medicine, Kobe University Graduate School of Medicine, Kobe, Japan

\section{Cardiovasc Diabetol 2016; 15: 79}

\section{Aims}

To study the effects of glycemic variability on neointimal growth after everolimus-eluting stent (EES) implantation.

\section{Methods}

Fifty patients all on statins ( 29 with known diabetes on diet only; 21 with no diabetes) who were undergoing EES implantation had continuous glucose monitoring before stenting to determine baseline glycemic variability as measured by the mean amplitude of glycemic excursion (MAGE). At 9 month follow-up, optical coherence tomography (OCT) was used to calculate the mean neointimal thickness (NIT) within 360 equally spaced radial sectors for every $1 \mathrm{~mm}$ cross-sectional OCT analysis. Major adverse cardiovascular events (MACE) were also assessed at 9 month follow-up.

\section{Results}

Sixty lesions in 50 patients were evaluated at 9 month follow-up. Univariate analysis showed that MAGE was most strongly correlated with NIT measurements (coefficient $\beta \pm$ 
standard error $=0.267 \pm 0.073$ and $0.016 \pm 0.003, \mathrm{t}=3.668$ and 6.092 , both $P<0.001$, respectively). In multivariate analysis, MAGE had the strongest effect on variability in NIT (coefficient $\beta \pm$ standard error $=0.239 \pm 0.093, P=0.014$ ) and the percentage of uncovered struts (coefficient $\beta \pm$ standard error $=0.019 \pm 0.004, P<0.001)$. Five lesions in four patients required target lesion revascularization (10.0\%). Compared to non-MACE cases, cases of MACE exhibited a significantly higher MAGE (99 vs. 68; $P=0.004)$, maximum NIT (580 vs. $330 \mu \mathrm{m} ; P=0.002)$, and variability in NIT (100 vs. 65 ; $P=0.007)$. HbA1c levels were not significantly different in these two groups.

\section{Conclusions}

Glycemic variability as measured by MAGE using CGM may affect vessel healing after drug eluting stent implantation in patients with coronary artery disease (CAD). Decreasing glycemic variability regardless of their HbAlc may be an important target for secondary prevention after coronary stenting, which is independent of dyslipidemia control.

\section{Comment}

Patients with diabetes and known CAD have a high risk of restenosis when undergoing percutaneous coronary intervention (PCI). Drug eluting stents such as EES have decreased the rate of restenosis but the presence of diabetes is still associated with restenosis and poor cardiovascular (CV) outcomes. Several studies have shown that glucose variability, regardless of diabetes status, can provoke oxidative stress that leads to endothelial cell dysfunction, progression of coronary atherosclerosis, and plaque vulnerability. In this study (14), glycemic variability as measured by MAGE was correlated with distorted neointimal growth as well as incomplete neointimal coverage of the stented vessel wall. In patients with MACE compared to non-MACE, there was a higher incidence of delayed vessel healing which was accompanied by higher glycemic variability regardless of the A1c levels.

\section{Glucose variability in a 26-week randomized comparison of mealtime treatment with rapid- acting insulin versus GLP-1 agonist in participants with type 2 diabetes at high cardiovascular risk}

\author{
Jeffrey L. Probstfield ${ }^{l}$, Irl B. Hirsch ${ }^{l}$, Barry R. Davis ${ }^{2}$, \\ Andrew Ahmann ${ }^{3}$, Richard Bergenstal ${ }^{4}$, Matthew Gilbert ${ }^{5}$, \\ Connie Kingry', Dorrine Khakpourl, Dejian Lai ${ }^{2}$, \\ Sarah L. Pressel ${ }^{2}$, Kelley R. Branch ${ }^{1}$, Matthew Riddle ${ }^{3}$, \\ Kevin D. O'Brien' ${ }^{1}$ on behalf of the FLAT-SUGAR Trial \\ Investigators \\ ${ }^{1}$ University of Washington, Seattle, WA; ${ }^{2}$ The University of \\ Texas Health Science Center, Houston, TX; ${ }^{3}$ Oregon Health \\ \& Science University, OR; ${ }^{4}$ Park Nicollet, Minneapolis, \\ $M N ;{ }^{5}$ University of Vermont, Burlington, VT
}

Diabetes Care 2016; 39: 973-981

\section{Aims}

This study, known as the FLuctuATion reduction with inSUlin and glucagon-like peptide (GLP-1) Added togetheR
(FLAT-SUGAR) study, was designed as a proof-of-concept study to determine whether glycemic variability $(\mathrm{GV})$ and cardio-metabolic risk markers can be decreased more by meal-time therapy with a short-acting GLP-1 agonist than with a rapid-acting insulin analog while A1c levels are kept equivalent in people with insulin-requiring type 2 diabetes and high cardiovascular risk.

\section{Methods}

After an 8 to 12 week run-in on metformin and basal-bolus insulin (BBI), 102 participants were randomized to exenatide dosing before the two largest meals instead of meal-time insulin (GLIPULIN cohort) or continuation of rapid-acting insulin analogs (BBI cohort). Measurements of $\mathrm{GV}$ by continuous glucose monitoring (CGM), hypoglycemia, weight, cardiometabolic risk markers, and cardiac arrhythmias were assessed. The primary end point was change in glucose coefficients of variation $(\mathrm{CV})$ by $\mathrm{CGM}$ from baseline to 26 weeks.

\section{Results}

At randomization, the median $\mathrm{A} 1 \mathrm{C}$ were $7.3 \%$ and $7.4 \%$ and the glucose CVs were 30.3 and 31.9 for BBI and GLIPULIN cohorts, respectively. At 26 weeks, A1C levels were similar $(7.1 \%$ vs. $7.2 \%)$ whereas mean $\mathrm{CV}$ improved $(-2.4$ vs. $0.4, P=0.047$ ) favoring the GLIPULIN cohort. Other GV indices (SD, IQR, MAGE, continuous overall net glycemic action [CONGA], mean of daily differences [MODD]) followed similar nonsignificant patterns of improvement with the GLIPULIN cohort. There were no differences in hypoglycemic events during CGM or arrhythmias during electrocardiographic monitoring. Changes in body weight $(-4.8 \mathrm{~kg}$ vs. $+0.7 \mathrm{~kg}, P<0.001)$, alanine aminotransferase $(P=0.0002)$, and serum amyloid A $(P=0.023)$ favored the GLIPULIN cohort but other cardio-metabolic risk markers (albuminuria, C-reactive protein (CRP), interleukin 6, and urinary isoprostanes) did not change.

\section{Conclusions}

In people with insulin-requiring diabetes and high-risk cardiovascular risk, the short-acting GLP-1 agonist group compared to the rapid-acting insulin group reduced $\mathrm{GV}$, weight, and some cardio-metabolic risk markers while maintaining equivalent A1c levels.

\section{Comments}

$\mathrm{HbA1c}$ is associated with long-term diabetes complications but does not reflect glycemic variability that may worsen outcomes by oxidative stress, inflammation, and cardiac arrhythmias. The goal of the FLAT-SUGAR study (15) was to show GV and cardio-metabolic risk markers may be decreased with no significant change in $\mathrm{HbA1c}$ levels in insulin-requiring patients with high $\mathrm{CV}$ risk. As expected, in the short-acting GLP-1 group, GV was significantly reduced with a reduction in weight and two cardio-metabolic risk markers (alanine aminotransferase and serum amyloid A). Other cardio-metabolic risk factors as well as arrhythmias were not changed, most likely due to no differences in hypoglycemia or marked changes in glycemic variability. 
The LEADER study (16) (Liraglutide Effect and Action in Diabetes: Evaluation of Cardiovascular Outcome Results) showed a significant, $13 \%$, reduction in the primary three-point MACE (non-fatal myocardial infarction (MI), non-fatal stroke, and CV death) with a $22 \%$ reduction in $\mathrm{CV}$ death. These outcomes could be driven by a $31 \%$ lower rate of severe hypoglycemia and a $20 \%$ lower rate of the combination of severe and confirmed hypoglycemia (plasma glucose level, <56 mg per deciliter) in the liraglutide group than in the placebo group. In the ELIXA trial (17) (Evaluation of Lixisenatide in Acute Coronary Syndrome), the GLP-1-receptor agonist lixisenatide, which is shorter-acting than and structurally dissimilar to liraglutide, did not show any cardiovascular benefit in patients with diabetes and a recent acute coronary syndrome (ACS). The study cohort being ACS and no difference in hypoglycemia between these two groups could drive the lack of $\mathrm{CV}$ benefit in the ELIXA trial.

\section{Conclusion}

Like in so many clinical paradigm shifts in the history of medicine, the routine use of CGM gets its fair portion of skepticism, ignorance, and overt rejection. This is finally all good: diabetes is a chronic disease that puts huge burden onto every individual that has to deal with it - as a patient, a close relative/partner of a patient, or a health-care professional, and all treatment modalities so far are not even close to ideal. Initial fierce criticism of CGM technologies from professionals was out-weighed by the immense hope of people with diabetes. The CGM maturation process, that we try to follow, develops towards a new outcome measure: the glucose variability.

But what does it mean for the patient (18)? The wellbeing of patients (19) and their families (20) is determined mostly by the disease burden. Is this also a new outcome measure? CGM can clearly help patients improving their metabolic control and increase the time blood glucose stays in the target range (21), but it is the automation of diabetes management with closed-loop insulin delivery in an artificial pancreas that will finally reduce the disease burden. The evidence rendering glucose variability dangerous is accumulating and the time within the range will likely become a major endpoint; it is high time to engage into pivotal clinical trials with the advanced technologies and finally give to people with diabetes peace of mind.

\section{Author Disclosure Statement}

B. B. receives consultancy fees from Medtronic. B. B. has interest in Medtronic, Dexcom, and Abbott with research and grant support to his employer.

T. B. served on advisory boards of Novo Nordisk, Sanofi, Eli Lilly, Boehringer, Medtronic, and Bayer Health Care. T. B's institute received research grant support, with receipt of travel and accommodation expenses in some cases, from Abbott, Medtronic, Novo Nordisk, GluSense, Sanofi, Sandoz, and Diamyd. T. B. received honoraria for participating on the speaker's bureaux of Eli Lilly, Bayer, Novo
Nordisk, Medtronic, Sanofi, and Roch. T. B. owns stocks of DreamMed.

\section{References}

1. Kovatchev B, Cobelli C. Glucose variability: Timing, risk analysis, and relationship to hypoglycemia in diabetes. Diabetes Care 2016; 39: 502-10.

2. Hirsch IB. Glycemic variability and diabetes complications: does it matter? Of course it does! Diabetes Care 2015; 38: $1610-14$.

3. Bergenstal RM. Glycemic variability and diabetes complications: does it matter? Simply put, there are better glycemic markers! Diabetes Care 2015; 38: 1615-21.

4. Foster NC, Miller KM, Tamborlane WV, Bergenstal RM, Beck RW; T1D Exchange Clinic Network. Continuous glucose monitoring in patients with type 1 diabetes using insulin injections. Diabetes Care 2016; 39: e81-2.

5. Juvenile Diabetes Research Foundation Continuous Glucose Monitoring Study Group, Tamborlane WV, Beck RW, Bode BW, Buckingham B, Chase HP, Clemons R, FialloScharer R, Fox LA, Gilliam LK, Hirsch IB, Huang ES, Kollman C, Kowalski AJ, Laffel L, Lawrence JM, Lee J, Mauras N, O’Grady M, Ruedy KJ, Tansey M, Tsalikian E, Weinzimer S, Wilson DM, Wolpert H, Wysocki T, Xing D. Continuous glucose monitoring and intensive treatment of type 1 diabetes. $N$ Engl J Med 2008; 359: 1464-76.

6. Lind M, Polonsky W, Hirsch IB, Heise T, Bolinder J, Dahlqvist S, Pehrsson NG, Moström P. Design and methods of a randomized trial of continuous glucose monitoring in persons with type 1 diabetes with impaired glycemic control treated with multiple daily insulin injections (GOLD Study). J Diabetes Sci Technol 2016; 10:754-61.

7. Bachmann S, Hess M, Martin-Diener E, Denhaerynck K, Zumsteg U. Nocturnal Hypoglycemia and physical activity in children with diabetes: new insights by continuous glucose monitoring and accelerometry. Diabetes Care 2016; 39: e95-6.

8. Karges B, Kapellen T, Wagner VM, Steigleder-Schweiger C, Karges W, Holl RW, Rosenbauer J; DPV Initiative. Glycated hemoglobin A1c as a risk factor for severe hypoglycemia in pediatric type 1 diabetes. Pediatr Diabetes 2015; Dec 29. doi:10.1111/pedi.12348. [Epub ahead of print]

9. Bratina N, Shalitin S, Phillip M, Battelino T. Type 1 Diabetes in the young: Organization of two national centers in Israel and Slovenia. Zdr Varst 2015; 54: 139-45.

10. Pettus J, Edelman SV. Use of glucose rate of change arrows to adjust insulin therapy among individuals with type 1 diabetes who use continuous glucose monitoring. Diabetes Technol Ther 2016; 18 Suppl 2: S234-42.

11. Battelino T, Omladič JŠ, Phillip M. Closed loop insulin delivery in diabetes. Best Pract Res Clin Endocrinol Metab 2015; 29: 315-25.

12. Choudhary P, Olsen BS, Conget I, Welsh JB, Vorrink L, Shin JJ. Hypoglycemia prevention and user acceptance of an insulin pump system with predictive low glucose management. Diabetes Technol Ther 2016; 18: 288-91.

13. Malone JI. Diabetic Central Neuropathy: CNS damage related to hyperglycemia. Diabetes 2016; 65: 355-7.

14. Kuroda M, Shinke T, Otake H, Sugiyama D, Takaya T, Takahashi H, Terashita D, Uzu K, Tahara N, Kashiwagi D, Kuroda K, Shinkura Y, Nagasawa Y, Sakaguchi K, Hirota Y, Ogawa W, Hirata K. Effects of daily glucose fluctuations on the healing response to everolimus-eluting stent 
implantation as assessed using continuous glucose monitoring and optical coherence tomography. Cardiovasc Diabetol 2016; 15: 79.

15. FLAT-SUGAR Trial Investigators. Glucose variability in a 26-Week randomized comparison of mealtime treatment with rapid-acting insulin versus GLP-1 agonist in participants with type 2 diabetes at high cardiovascular risk. Diabetes Care 2016; 39: 973-81.

16. Marso SP, Daniels GH, Brown-Frandsen K, Kristensen P, Mann JF, Nauck MA, Nissen SE, Pocock S, Poulter NR, Ravn LS, Steinberg WM, Stockner M, Zinman B, Bergenstal RM, Buse JB; LEADER Steering Committee; LEADER Trial Investigators. Liraglutide and cardiovascular outcomes in type 2 diabetes. $N$ Engl J Med 2016; 375: 311-22.

17. Pfeffer MA, Claggett B, Diaz R, Dickstein K, Gerstein HC, Køber LV, Lawson FC, Ping L, Wei X, Lewis EF, Mag- gioni AP, McMurray JJ, Probstfield JL, Riddle MC, Solomon SD, Tardif JC; ELIXA Investigators. Lixisenatide in patients with type 2 diabetes and acute coronary syndrome. $N$ Engl J Med 2015; 373: 2247-57.

18. Rodbard D. Continuous Glucose Monitoring: A review of successes, challenges, and opportunities. Diabetes Technol Ther 2016; 18 Suppl 2: S23-213.

19. Klemenčič S, de Wit M, Rutar M, Battelino T, Bratina N. Annual psychological screening in youth and young adults with type 1 diabetes. Zdr Varst 2015; 54: 103-11.

20. Pate T, Klemenčič S, Battelino T, Bratina N. Fear of hypoglycemia, anxiety, and subjective well-being in parents of children and adolescents with type 1 diabetes. $J$ Health Psychol 2016; Jun 7. [Epub ahead of print] pii: 1359105316650931

21. Dovč K, Bratina N, Battelino T. A new horizon for glucose monitoring. Horm Res Paediatr 2015; 83: 149-56. 